\title{
Experiments in Planar Haptic Contour Exploration
}

\author{
Kee-Yip Chan Kelly Kitagawa Roberto Manduchi \\ University of California, Santa Cruz \\ E-mail:kyc@soe.ucsc.edu,kkitagaw@ucsc.edu,manduchi@soe.ucsc.edu
}

\begin{abstract}
We present some experimental results concerning exploration of planar contours (more specifically, polygons) using a 2-D haptic device, the Logitech Wingman mouse. With respect to previous work, our project focused on improving the contour tracing experience using the effects provided by the Immersion TouchSense API.
\end{abstract}

\section{Introduction and Previous Work}

In this short paper we present some experimental results concerning exploration of planar contours (more specifically, polygons) using a 2-D haptic device, the Logitech Wingman mouse ${ }^{1}$. Haptic devices have been proposed as a viable tool to give a blind person access to graphical information. As noted in [4], multimodal (haptic + audio) interfaces have a number of advantages with respect to traditional tactile diagrams, including short production time, ease of changing/editing, and the possibility of interactive and adaptive behaviors. The possibilities of 2-D haptics for the education of visually impaired children have also been studied in [3]. A comparison of the Wingman mouse with PHANToM, a popular (but expensive) 3-D device, was presented in [5]. Experiments with exploration of a bar graph have shown that the latter has better performances when used alone, but that that the two perform similarly when sound feedback is involved. Preliminary experiments using the Wingman mouse for simple shape exploration have also been described in [1]. Other projects (e.g. [2]) have used the Wingman mouse but without exploiting its full force-feedback capabilities.

With respect to previous work, our project focused on improving the contour tracing experience by making diligent use of the effects provided by the Immersion TouchSense API. As noted in [6], simple application of basic effects (such as "groove", "wall" or "texture") creates serious difficulties in the case of corners. Our improved design for corners simplifies exploration by increasing ease

\footnotetext{
${ }^{1}$ The Wingman mouse is currently out of production, although it is still available in the market.
}

of transitioning from one side of the polygons to the next one.

\section{Design}

We simulate the edges of a polygon using tactile groove effects. The out-of-box groove effect provided by the Immersion IFC SDK is insufficient for our needs because its tactile force is far too weak, and may let the user exit the groove too easily. Fortunately, we found that layering four of these grooves resulted in a much stronger effect, and therefore is more effective in keeping the user's cursor within the constraints of the edge. However, this enhanced groove alone is not enough for a satisfactory tactile exploration of the polygon's shape.

To minimize ambiguity at the corners, only a single 4layer groove is activated at a time, based on the shortest distance to the user's cursor. But because any effect is also disabled when the cursor moves outside of its area, it becomes far too easy for the cursor to move past a corner. To remedy this, we added an attractor at each corner, where the attractor can be activated jointly with the groove. Still, with this design the user needs to probe ambiguously for the next edge because the resistive force of the attractor towards its center provides no hints on the direction/location of the edges. Finally, the solution that yielded satisfactory maneuverability is to disable the attractor if the cursor is within the inner region of the polygon. The user is then able to recognize that moving in the direction absent of resistive forces will lead to the next edge of the polygon. In addition, the fact that the two 4-layer groove effects meet at the corner helps to prevent the cursor from veering inside the polygon. To reinforce the policy of not moving inside the polygon, the attractor is activated if the cursor is at a distance greater than half the attractor's radius.

Acoustic interface is also provided. Only when the cursor is in a groove there is no sound. Different sounds are enabled when the cursor enters a"forbidden" area (the interior of a polygon), when the cursor is at a corner, when the cursor hits the edge of the current work area, and when the cursor is outside the polygon (with a pitch that changes depending on the distance to the polygon's contour) 


\section{Experiments}

We have ran a number of experiments with 14 users, all students (undergraduate and graduate) in different disciplines at UCSC. The subjects were blindfolded during the experiment, except when asked to draw a shape (see later). Each user underwent a "training" phase using the simple triangular shape of Fig. 1(a). The haptic effects and sounds were explained to the user, who was allowed to practice with the system taking as much time as he or she wanted. Then the user was tested on the three shapes of Fig. 1(b-d). For each shape, the user was asked to interact with it until he or she felt that they had formed an idea of how the shape is made. The elapsed time was recorded. At the end of the exploration, the user was asked how many vertices were in the polygon. Then, the blindfold was removed from the user and he or she was asked to draw a sketch of the polygon. Note that the users were informed in the beginning of the experiment that the shapes were all polygonals, and that they will be asked to draw the shapes after interacting with the system. Finally, the user was asked a few questions about his or her experience with the device.

Most users felt that sound feedback was indeed effective, and that it was rather difficult to fall outside the shape while tracking its contour. The users were evenly divided about whether they had trouble moving to the next corner, and about whether the were comfortable with the mouse effect. Overall, the users rated their overall experience fairly positive. Other comments from the users highlighted the difficult they had in conveying their mental image of the polygon to a drawing. This is substantiated by the numerical results reported below.

The average elapsed time for exploration of the three shapes was higher than expected (106, 257 and $226 \mathrm{sec}-$ onds respectively). We conjecture that experience and training may reduce the amount of time required for learning the shape. Indeed, note that exploration of the last and most complex shape (Fig. 1(d)) required less time on average than exploration of the simpler shape of Fig. 1(c), which was performed earlier.

For the quadrilateral of Fig. 1(b), all user correctly derived the shape and the number of vertices. However, the correct number of vertices was detected only by 4 users for the case of Fig. 1(c) and by 6 users for the case of Fig. 1(d). In general, users tended to underestimate the number of vertices by 1 or 2 in these last two cases. In order to quantify the similarity between the drawn and the actual shape, we looked for evidence of "geometric coherence". Basically, we checked each drawn sketch to see whether the user was able to infer the presence of a single concavity (Fig. 1(c)) or of two consecutive concavities (Fig. 1(d)). The number of users who identified the correct number of concavities in the two cases were 6 and 9 respectively.

These simple experiments show that, even when the user can correctly trace the polygon's contour with the hap- tic mouse, building a correct mental image from a 2-D haptic map may prove challenging. Further investigation is needed in order to assess the ability of individuals (and in particular, of blind individuals) to learn and memorize spatial schemes and relationships based on 2-D haptic maps.

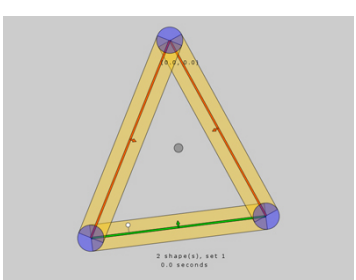

(a)

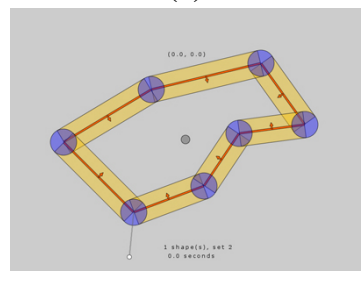

(c)

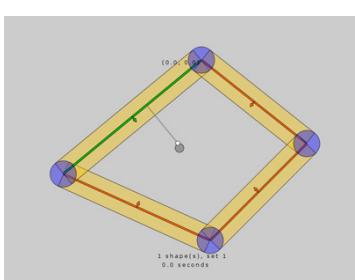

(b)

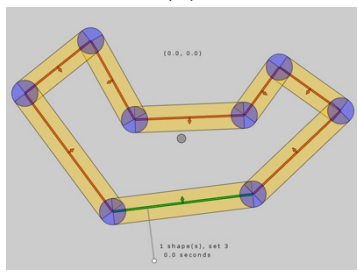

(d)
Figure 1: The polygon used for training (a) and the three polygon used for testing.

\section{Acknowldegments}

This work was supported by a Special Research Grant from the Committee on Research at UCSC.

\section{References}

[1] R. G. Golledge, M. Rice, and R. D. Jacobson. A commentary on the use of touch for accessing on-screen spatial representations: The process of experiencing haptic maps and graphics. The Professional Geographer, 57(3):339-349, 2005.

[2] P. Parente and G. Bishop. BATS: The blind audio tactile mapping system. In Proceedings of the ACM Southeast regional conference, Savannah, GA, 2003.

[3] W. Wies, J. Gardner, M. O'Modhrain, C. Hasser, and V. Bulatov. Web-based touch display for accessible science education. In Proceedings of the First International Workshop on Haptic Human-Computer Interaction, pages 52-60, London, UK, 2001.

[4] W. Yu and S. Brewster. Multimodal virtual reality versus printed medium in visualization for blind people. In Assets '02: Proceedings of the 5th International ACM Conference on Assistive Technologies, pages 57-64, 2002.

[5] W. Yu and S. Brewster. Evaluation of multimodal graphs for blind people. Universal Access in the Information Society, 2(2):105-24, 2003.

[6] W. Yu, K. Guffie, and S. Brewster. Image to haptic data conversion: A first step to improving blind peoples accessibility to printed graphs. In Proc. of Eurohaptics, 2001. 